\title{
Exposure of haptic of posterior chamber intraocular lens after sutureless intrascleral fixation
}

\author{
Yoshitsugu Matsui ${ }^{*}$, Hisashi Matsubara$^{1}$, Tsukasa Hanemoto $^{2}$ and Mineo Kondo ${ }^{1}$
}

\begin{abstract}
Background: A technique of sutureless intrascleral fixation of an intraocular lens (IOL) in an eye that lacks a posterior capsular support has been reported. The advantage of this technique was that the suture-related complications did not develop. However, the long-term complications of a sutureless IOL implantation have not been reported.

Case presentation: A 75-years-old man had a sutureless intrascleral fixation (Y-fixation) of an IOL 4 months before our examination. The nasal haptic became exposed and the temporal haptic was seen in the subconjunctiva. The tilted IOL was removed and replaced by a posterior chamber IOL that was sutured to the sclera. At the 6 months examination, the eye was quiet and the IOL was stable.

Conclusion: We suggest that the exposure of the nasal haptic of an IOL that was implanted by sutureless intrascleral fixation (Y-fixation) was due to poor surgical technique and/or the erosion of a fragile sclera. Thus, eyes should be carefully and frequently monitored after sutureless intrascleral posterior chamber IOL implantation.
\end{abstract}

\section{Background}

An implantation of an intraocular lens (IOL) in eyes that lack a posterior capsular support has been accomplished with an iris-fixed IOL, an anterior chamber IOL, or a transscleral IOL fixed by sutures passed through the ciliary sulcus or pars plana. In 2006, Gabor introduced a transscleral needle fixation of an IOL [1], and in 2007, Agarwal introduced the glued IOL technique in which the intrascleral haptic of a posterior chamber IOL is glued to the sclera [2]. In 2012, Ohta introduced the Y-fixation technique that fixed an IOL without large lamellar scleral flaps and fibrin glue [3].

The advantage of these techniques is that it avoided suturing and thus the suture-related complications, and it required no specially designed haptics. The sutureless intrascleral fixation technique allowed an exact centration of the IOL and provided axial stability $[4,5]$. However, there are only a few long-term follow-up studies on the complications of these techniques. We report a case in

\footnotetext{
* Correspondence: footboyslim366@gmail.com

'Department of Ophthalmology, Mie University Graduate School of Medicine, 2-174 Edobashi, Tsu, Mie 514-8507, Japan

Full list of author information is available at the end of the article
}

which the IOL haptic became exposed three months after the IOL was implanted with the Y-fixation technique.

\section{Case presentation}

A 75-year-old man was referred to our hospital with an exposed IOL haptic. He had undergone lensectomy for a cataract in his left eye 30-years earlier but no surgical details were available. He then had an extracapsular IOL implantation 20-years earlier and the out-of-the-bag IOL dislocated 1-year ago. He then had another IOL (NX-70, Santen, Japan) implanted with the Y-fixation technique.

Three months postoperatively, the nasal haptic of the IOL haptic was found to be exposed and a self-scleral patch was grafted to cover the haptic at the previous hospital. However, the haptic became exposed again 1 month after the surgery, and he was referred to our hospital with a 1-month history of exposed IOL haptic in his left eye.

Our examination showed that his decimal bestcorrected visual acuity (BCVA) was 0.5 with a tilted IOL. The nasal IOL haptic was exposed and the temporal haptic could be seen in the subconjunctiva (Fig. 1a-c). To correct this condition, the conjunctiva around the haptics was opened, and the both haptics 

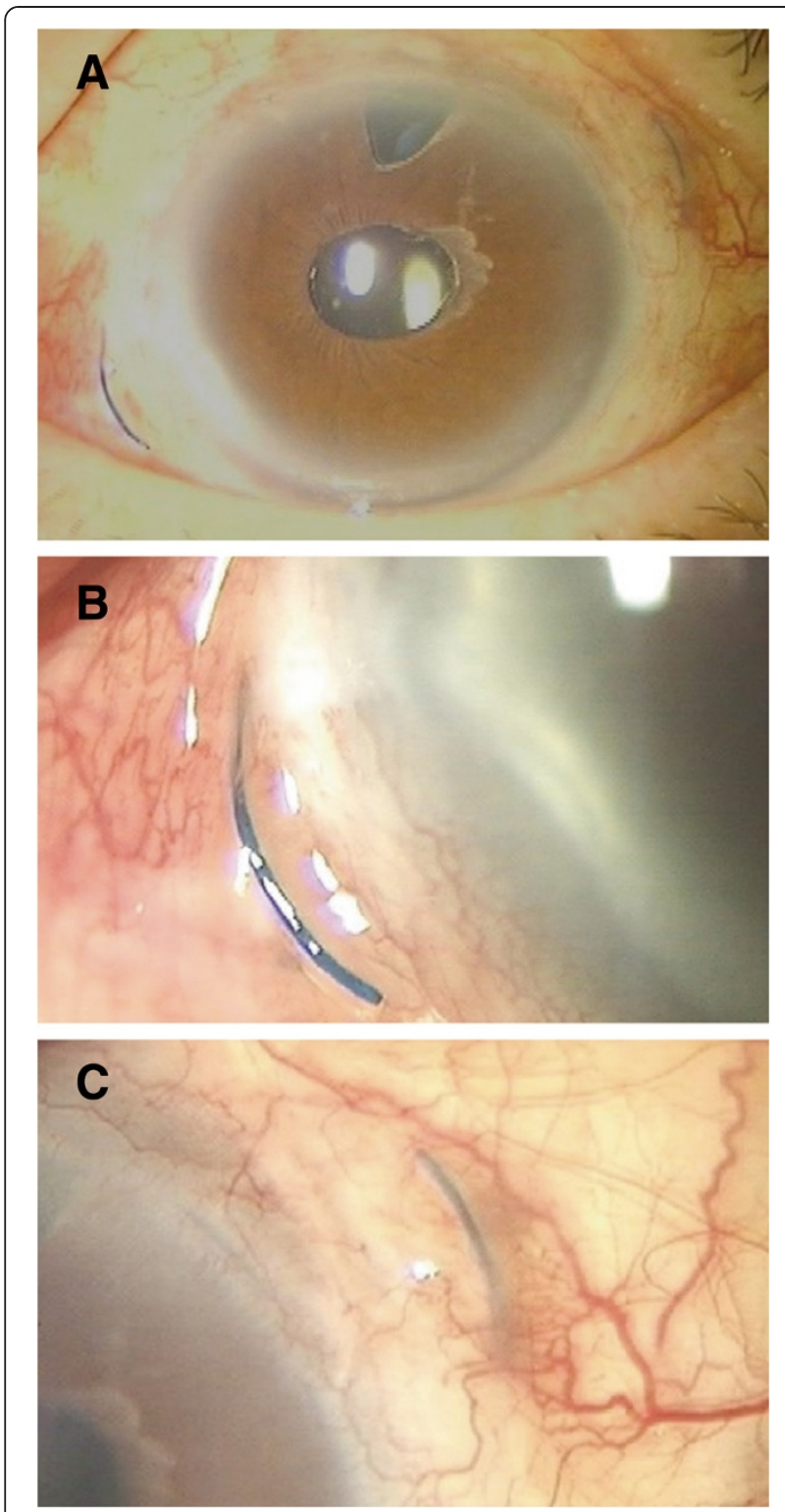

Fig. 1 Photograph of the anterior segment of the eye showing an exposed haptic of an intraocular lens (IOL). a Preoperative view of the anterior segment. $\mathbf{b}$ The nasal haptic of an intraocular lens (IOL) is visible. c The temporal haptic can be seen in the subconjunctiva

exposed. The Y-shaped incision and scleral flap were not detected. The tilted IOL was removed and a posterior chamber IOL was implanted and sutured.

At the 6 months follow-up examination, the eye was stable and the BCVA was 1.0. The IOL was fixed and aligned.

\section{Discussion}

As best we know, another case of haptic exposure after sutureless Y-fixation has not been published. There are several possible explanation for the exposure of the nasal haptic. One is that a scleral tunnel was not constructed correctly. This procedure requires the creation of the scleral tunnel parallel to the limbus at the branching point of a Y-shaped incision. The scleral tunnel must be thick enough to prevent the protrusion of the haptic. Kumar has reported that the depth of the tunnel can alter the degree of scleral erosion by the haptic, and a subconjunctival haptic exposure was seen in three eyes (1.4 \%) with thin scleral flaps [6]. Ohta recommended a 24-gauge MVR knife be used to create the sclerotomy for the appropriate haptic fixation instead of a needle [7]. It is easy to create an incision having a uniform thickness sclera with this knife. Ohta also recommended a single 8-0 nylon suture should be used to fix the haptic to the scleral bed to prevent it from shifting after surgery. In our case, the single 8-0 nylon suture approach was used in previous surgery at another hospital, but the haptic became malpositioned after 3 month of the Y-fixation technique.

A second factor for the haptics exposure is that an IOL specifically designed for sutureless intrascleral posterior chamber IOL implantation is not available. However, Gabor stated there is no need for special IOL designs for this method even though it is not known whether the structure of the sclera can be maintained for a long time. The average diameter of the cornea in Japanese is 11.5 to $12 \mathrm{~mm}$, and the Y-shaped incision is made $2.0 \mathrm{~mm}$ from the limbus. Therefore, the full diameter becomes approximately $14 \mathrm{~mm}$ for the scleral fixation. However, an IOL is designed for intracapsular fixation, and the full length of an IOL with an optic diameter of $7.0 \mathrm{~mm}$ IOL is $13.5 \mathrm{~mm}$ in the bag. When this curved haptic is inserted in a linear sclera tunnel, an unnatural tension is placed on the sclera. This may cause torsion of the IOL and erosion of the sclera (Fig. 2). Kumar reviewed the complications in 486 eyes that had a single-piece PMMA IOL with an optic diameter of $6.5 \mathrm{~mm}$ implanted. The IOL haptic-related complication were; haptic displacement (4.1\%), haptic tip

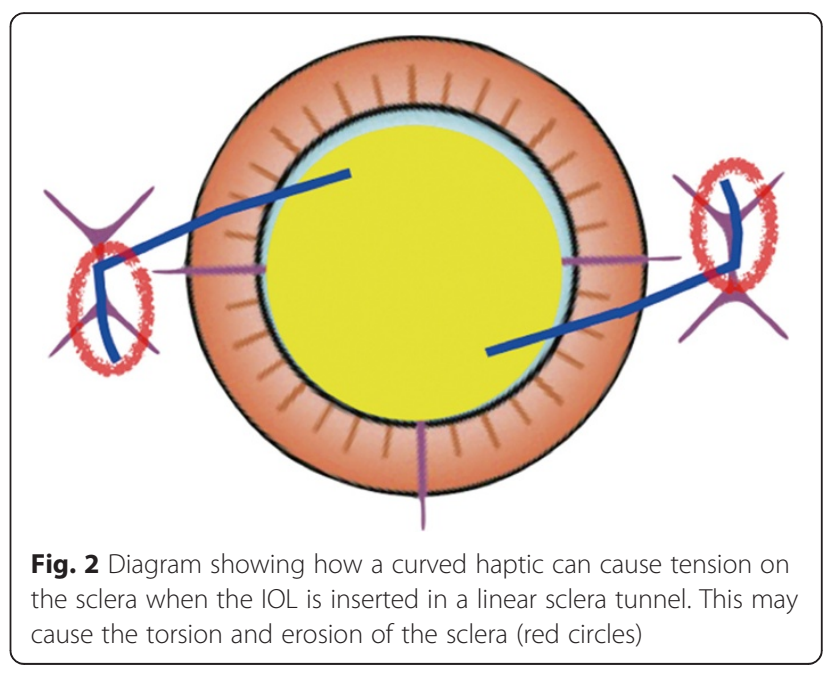


extrusion (0.8\%), subconjunctival haptic (0.4\%), and no exposed IOL haptic [8]. In addition, the incidence of exposed suture knots through the conjunctiva was 3.8 to $11 \%$ in the late postoperative period of suture-assisted scleral fixated IOL implantations [9-12].

Both an exposed IOL haptic and exposed sutures are potential risks of endophthalmitis. However, long-term follow-up studies are lacking, and further studies comparing the structure of the sclera and the status of the sutures and haptic need to be conducted.

We recommend that the fragility of the sclera be estimated preoperatively. In our case, a self-scleral patch was grafted to cover the haptic 3 months after the Y-fixation technique surgery, but 1 month after the surgery the haptic was exposed again. This suggested that our patient had relatively fragile sclera. The patient did not have a medical history of inflammation such as scleritis, episcleritis, rheumatoid arthritis, and herpes zoster ophthalmicus, but had a history of two cataract surgeries. Many cases of sutureless intrascleral posterior chamber IOL implantation may be a second implantation surgery that had had scleral incisions. In addition, damage of the sclera may have occurred in the preoperatively from aggressive thermo-coagulation hemostasis of the sclera and insufficient controlling inflammation. Thus, when the Y-fixation technique is used, it is important to avoid damage to the sclera to maintain the integrity of the sclera for the implantation surgery.

\section{Conclusions}

In conclusion, we have presented our findings in a case of an exposed haptic of an IOL after implantation with the sutureless Y-fixation technique. We recommend that the surgeon create a scleral tunnel correctly, and the sclera be carefully examined to determine if there are any signs of fragility before this procedure is used.

\section{Consent}

The patient was fully informed about the examinations and provided written consent. Written consent was obtained from the patient for publication of this case report and any accompanying images. A copy of the written consent is available for review by the Editor.

\section{Competing interests}

The authors declare that they have no competing interests.

\section{Authors' contributions}

YM: patient interaction, performed surgery, literature research, drafting and final approval of manuscript. HM: patient interaction, patient diagnosis, performed surgery, critical revision, final approval of manuscript. TH: critical revision and final approval of manuscript. MK: critical revision, language editing and final approval of manuscript. All authors read and approved the final manuscript.

\section{Acknowledgement}

We thank Professor Duco Hamasaki of the Bascom Palmer Eye Institute of the University of Miami for critical discussion and final manuscript revisions.

\section{Author details}

${ }^{1}$ Department of Ophthalmology, Mie University Graduate School of Medicine, 2-174 Edobashi, Tsu, Mie 514-8507, Japan. Kozawa Eye Hospital, Ibaragi, Japan.

Received: 14 April 2015 Accepted: 6 August 2015

Published online: 14 August 2015

\section{References}

1. Gabor SGB, Pavilidis MM. Sutureless intrascleral posterior chamber intraocular lens fixation. J Cataract Refract Surg. 2007;33(11):1851-4.

2. Agarwal A, Kumar DA, Jacob S, Baid C, Agarwal A, Srinvasan S. Fibrin glue-assisted sutureless posterior chamber intraocular lens implantation in eyes with deficient posterior capsules. J Cataract Refractive Surg. 2008;34(9):1433-8.

3. Ohta T. Y-fixation technique. In: Agarwal A, editor. Glued IOL. New Delhi: Jaypee Brothers Med Pub; 2013. p. 88-96.

4. Kumar DA, Agarwal A, Agarwal A, Chandrasekar R, Priyanka V. Long-term assessment of tilt of glued intraocular lenses: an optical coherence tomography analysis 5 years after surgery. Ophthalmology. 2015;122(1):48-55.

5. Yamane S, Inoue M, Arakawa A, Kadonosono K. Sutureless 27-gauge needle-guided intrascleral intraocular lens implantation with lamellar scleral dissection. Opthalmology. 2014;121(1):61-6.

6. Kumar DA, Agarwal A, Packiyalakshmi S, Jacob S, Agarwal A. Complications and visual outcomes after glued foldable intraocular lens implantation in eyes with inadequate capsules. J Cataract Refractive Surg. 2013;39(8):1211-8.

7. Ohta T, Toshida H, Murakami A. Simplified and safe method of sutureless intrascleral posterior chamber intraocular lens fixation: Y-fixation technique. J Cataract Refractive Surg. 2014;40(1):2-7.

8. Kumar DA, Agarwal A. Glued intraocular lens: a major review on surgical technique and results. Curr Opin Ophthalmol. 2013;24(1):21-9.

9. Genekal S, Venkataratnam S, Dorairaj S, Jhanji V. Comparative evaluation of suture-assisted and fibrin glue-assisted scleral fixated intraocular lens implantation. J Cataract Refracitve Surg. 2012;28(4):249-52.

10. Luk AS, Young AL, Cheng LL. Long-term outcome of scleral-fixated intraocular lens implantation. Br J Ophthalmol. 2013;97(10):1308-11.

11. Yang YF, Bunce C, Dart JKG, Johnston RL, Charteris DG. Scleral-fixated posterior chamber intraocular lenses in nonvitrectomised eyes. Eye. 2006;20:64-70

12. McAlister AS, Hirst LW. Visual outcomes and complications of scleral-fixated posterior chamber intraocular lenses. J Cataract Refractive Surg. 2011;37(7):1263-9.

\section{Submit your next manuscript to BioMed Central and take full advantage of:}

- Convenient online submission

- Thorough peer review

- No space constraints or color figure charges

- Immediate publication on acceptance

- Inclusion in PubMed, CAS, Scopus and Google Scholar

- Research which is freely available for redistribution 